\title{
Risk factors for retinal detachment following cataract surgery: the impact of posterior capsular rupture
}

\author{
Vasileios Petousis, ${ }^{1}$ Ahmed A Sallam, ${ }^{1}$ Richard J Haynes, ${ }^{2}$ C K Patel, ${ }^{3}$ Ajai K Tyagi, ${ }^{4}$ \\ James N Kirkpatrick, ${ }^{1}$ Robert L Johnston ${ }^{1}$
}

${ }^{1}$ Department of

Ophthalmology, Gloucestershire Hospitals NHS Foundation

Trust, Cheltenham, UK

${ }^{2}$ Vitreoretinal Unit, Bristol Eye

Hospital, Bristol, UK

${ }^{3}$ Department of

Ophthalmology, Oxford Radcliffe Hospitals NHS

Foundation Trust, Oxford, UK

${ }^{4}$ Department of

Ophthalmology, Sandwell and

West Birmingham Hospitals

NHS Trust, Birmingham, UK

Correspondence to

Robert L Johnston, Department of Ophthalmology, Cheltenham

General Hospital, Sandford

Road, Cheltenham,

Gloucestershire GL53 7AN, UK; rob.johnston@glos.nhs.uk

Received 29 August 2015 Revised 13 December 2015 Accepted 10 January 2016 Published Online First 8 February 2016

\section{CrossMark}

To cite: Petousis $\mathrm{V}$,

Sallam AA, Haynes RJ, et al. $\mathrm{Br} J$ Ophthalmol

2016:100:1461-1465.

\section{ABSTRACT}

Aim To investigate risk factors for retinal detachment (RD) after cataract surgery, particularly posterior capsular rupture (PCR) with or without vitreous loss.

Methods Single centre electronic medical record database study of 18065 consecutive first eye cataract operations performed between 2005 and 2014. Survival analysis was performed with Kaplan-Meier curves and a Cox proportional hazard regression analysis to calculate HRs with respect to RD.

Results The RD rate at 3 months and 7 years was $0.067 \%$ and $0.30 \%$, respectively, with a median time to RD of 15 months (mean: 18 months, range:

0-84 months). Men had a higher RD risk (HR 2.00; 95\%

Cl 1.03 to 3.88; $p=0.03$ ) in the univariate model. Patients $<60$ years and those $>80$ years had an HR of $5.12(95 \%$ $\mathrm{Cl} 2.60$ to $10.07 ; p<0.001)$ and $0.16(95 \% \mathrm{Cl} 0.38$ to $0.69 ; p=0.01$ ), respectively, compared with patients 60 80 years of age. Eyes longer than $25 \mathrm{~mm}$ had an HR of 3.98 (95\% Cl 1.93 to $8.20 ; p<0.001)$ compared with eyes 23-25 mm. PCR occurred in 400 (2.2\%) eyes. The HR for RD was 12.83 (95\% Cl 5.62 to 29.30; $p<0.001$ ) for $P C R$ with vitreous loss. There were no RD events in eyes with PCR without vitreous loss.

Conclusions The risk for RD after cataract surgery is higher in younger patients and eyes with longer axial length or PCR with vitreous loss during surgery.

\section{INTRODUCTION}

Retinal detachment (RD) following cataract surgery is rare $\left(0.16 \%-2.31 \%\right.$ at $7-10$ years $\left.^{1-6}\right)$ but is among the most common causes of severe visual loss after surgery. Despite the transition from extracapsular to phacoemulsification cataract extraction there is still a four to nine times increased risk of developing an RD 8-20 years after cataract extraction compared with age-matched phakic eyes. ${ }^{3}{ }^{4}$ Identified risk factors include: patient (male gender, younger age), eye (long axial length) and surgery-related factors (posterior capsular rupture (PCR), anterior vitrectomy). ${ }^{5-9}$

Few studies have analysed data from routine clinical practice and almost all previous studies included both eyes of patients in their analyses potentially biasing their results. The Royal College of Ophthalmologists in the UK has supported the development and implementation of detailed nationally agreed datasets within electronic medical record (EMR) systems including: the cataract national dataset (CND) and $\mathrm{RD}$ dataset to facilitate local and national audit. ${ }^{10-14}$ EMR systems that have implemented the CND mandate the recording of the presence or absence of specified complications during cataract surgery, facilitating detailed analysis of the relationship between specific operative complications and subsequent RD.

The purpose of this study was to estimate the contemporary incidence of $\mathrm{RD}$ after cataract surgery and to identify and quantify associated risk factors.

\section{PATIENTS AND METHODS}

All ophthalmic procedures in Gloucestershire since 2005 have been recorded at the point of care by the operating surgeon within an EMR system (Medisoft Ophthalmology, Medisoft, Leeds, UK) that collects all nationally agreed datasets. The presence or absence of operative complications is a compulsory field and is collected in $100 \%$ of cases. Surgeons choose from a predefined list of complications or select 'None' (see CND). ${ }^{10}$

Gloucestershire Hospitals National Health Service (NHS) Foundation Trust is the sole NHS provider of cataract surgery and is the only facility for retinal surgery (NHS or private) for a geographically discrete population, where few if any patients travel to seek surgery at neighbouring units but in order to maximise identification of subsequent $\mathrm{RD}$ cases the closest neighbouring units, Bristol Eye Hospital (40 miles (65 Km) away), Oxford Radcliffe Hospitals Foundation Trust (40 miles $(65 \mathrm{Km}))$ and Sandwell and West Birmingham Hospitals NHS Trust $(50$ miles $(80 \mathrm{Km}))$, which all use the same EMR were contacted and their databases searched for patients with a Gloucestershire postcode. For Birmingham Hospitals data were only available from 2008 onwards while search was extended to a second EMR also in use.

This study analysed all phacoemulsification operations performed from November 2005 to January 2014. It was conducted in accordance with the Declaration of Helsinki, and the UKs Data Protection Act and was approved as an audit by the audit committee of the trust, therefore no institutional review board/ethics committee approval was required.

Combined procedures, vitrectomised eyes and eyes with a history of trauma were excluded from the sample. All data were extracted from the EMR system using automated audit tools within the EMR. All eyes where an 'anterior vitrectomy' procedure was recorded as part of the cataract operation or where an operative complication that implied PCR with vitreous loss were classified as 'PCR-vitreous loss', regardless of whether this 
specific complication was ticked (as surgeons may have only record the more serious complication, for example: 'nuclear/epinuclear fragment into vitreous').

In order to avoid bias due to associations between eyes, only first operated eyes having cataract surgery were included in the statistical analysis. The data extraction included: gender, age, axial length, operative complications (in particular PCR and whether this occurred with or without vitreous loss) and time interval to RD. Continuous variables were stratified and transformed into categorical variables to facilitate statistical analysis. Age and axial length were both stratified into three comparably sized groups: $<60$ years, $60-80$ years, $>80$ years and $<23 \mathrm{~mm}$, $23-25 \mathrm{~mm}$ and $>25 \mathrm{~mm}$, respectively. The age groups were chosen based on the fact that the appearance of posterior vitreous detachment (PVD) rises dramatically after the age of 60 years and in order to facilitate comparison with related previous studies. $^{2} 715$ The calculation of the follow-up time was based on the assumption that all patients without an RD event and all patients where death was not recorded in the EMR were still under potential follow-up.

After presenting the descriptive statistics for the population studied, a survival analysis was performed with Kaplan-Meier curves used to present the results, with $\mathrm{RD}$ as the dependent variable. A Cox proportional hazard regression analysis, both univariate and multivariate, was used to calculate HRs with a CI of $95 \%$ for the studied risk factors. In the multivariate model, all factors were included initially and the variable with the higher $p$ value was sequentially removed (back stepwise) until only variables with $\mathrm{p}$ values $<0.05$ remained in the model. The statistical analysis was performed using SPSS V.22.0 (IBM SPSS Statistics), and figures were created using STATA V.13.0 (Stata, Texas, USA).

\section{RESULTS}

Between November 2005 and January 2014, 29429 phacoemulsification cataract operations were performed where the primary indication was cataract surgery only but 11364 'second' operated eyes were excluded, leaving 18065 first eye cataract surgery operations in 18065 patients available for analysis.

Table 1 Demographic characteristics according to gender

\begin{tabular}{lll}
\hline & Gender & \\
\cline { 2 - 3 } & $\begin{array}{l}\text { Female } \\
\text { No }(\%) \\
(\mathbf{n}=10535)\end{array}$ & $\begin{array}{l}\text { Male } \\
\text { No }(\%) \\
(\mathbf{n}=7530)\end{array}$ \\
\hline Age & & \\
$<60$ years & $762(7.2 \%)$ & $795(10.5 \%)$ \\
$60-80$ years & $5719(54.2 \%)$ & $4333(57.5 \%)$ \\
$>80$ years & $4054(38.4 \%)$ & $2402(31.8 \%)$ \\
Median age & 78.0 & 76 \\
Mean age & 76.3 & 74.4 \\
IQR & 12 & 13 \\
Axial length & & \\
$<23 \mathrm{~mm}$ & $4853(46.0 \%)$ & $1641(21.7 \%)$ \\
$23-25 \mathrm{~mm}$ & $4801(45.6 \%)$ & $4869(64.6 \%)$ \\
$>25 \mathrm{~mm}$ & $869(8.2 \%)$ & $1015(13.4 \%)$ \\
Median axial length & 23.1 & 23.7 \\
Mean axial length & 23.2 & 23.8 \\
IQR & 1.32 & 1.29 \\
\hline
\end{tabular}

The median age was 77 years with an IQR of 12 years. The median axial length was $23.3 \mathrm{~mm}$ with an IQR of $1.40 \mathrm{~mm}$ (table 1). By 3 months and by 7 years, 12 and 36 analysed eyes had developed an RD, a cumulative RD rate of $0.067 \%$ and $0.30 \%$, respectively (figure 1 ). Of the 36 eyes with $\mathrm{RD}, 4$ $(11.1 \%)$ were identified at the Bristol Eye Hospital, outside Gloucestershire. The search of the EMRs in Oxford and Birmingham yielded no additional cases. The median time to RD was 15 months (mean: 18 months, range: 0-84 months). Eighty-two $(0.45 \%)$ patients were registered as deceased during the postoperative period on the EMR.

\section{Patient-related risk factors}

The risk of RD among men was twice that of women (HR 2.00; $95 \%$ CI 1.03 to $3.88 ; \mathrm{p}=0.03$ ) but this difference was only statistically significant in the univariate model (figure 2A). Younger age conferred a higher risk of RD, with patients $<60$ years having a fivefold increased risk (HR 5.12; 95\% CI 2.60 to $10.07 ; \mathrm{p}<0.001)$ compared with the reference age group of 60 80 years and patients $>80$ years having an $84 \%$ reduced risk (HR 0.16 ; 95\% CI 0.38 to 0.69 ; $\mathrm{p}=0.01$ ) (figure $2 \mathrm{~B}$ ). This was true in both univariate and multivariate analyses (table 2 ).

\section{Eye-related and surgery-related risk factors}

Short eyes $(<23 \mathrm{~mm})$ did not have a statistically significantly different rate of $\mathrm{RD}$ compared with eyes of normal axial length (23-25 mm). Long eyes (>25 mm) had an almost fourfold higher risk in the univariate analysis (HR 3.98; 95\% CI 1.93 to $8.20 ; \mathrm{p}<0.001)$, reduced to 2.7 -fold in the multivariate analysis (HR 2.74; 95\% CI 1.31 to $5.71 ; \mathrm{p}<0.001$ ). Both results were statistically significant (figure 2C). HRs are shown in table 2.

PCR occurred in $400(2.2 \%)$ eyes of the $18065 ; 328(1.8 \%)$ with vitreous loss and $72(0.4 \%)$ without vitreous loss. Subsequent RD developed in: $29(0.16 \%)$ of the 17665 eyes with an intact posterior capsule, 7 (2.13\%) of the 328 eyes with PCR with vitreous loss and 0 of the 72 eyes with PCR without vitreous loss, during cataract surgery (table 2). PCR with vitreous loss, therefore, carried a 13-16-fold increased risk for RD (univariate model: HR $12.83 ; 95 \%$ CI 5.62 to $29.30 ; \mathrm{p}<0.001$, multivariate model: HR 15.94; 95\% CI 6.96 to 36.45; $\mathrm{p}<0.001)$. No eyes with PCR without vitreous loss developed an $\mathrm{RD}$ and hence statistical analysis was not applicable (figure 2D).



Figure1 Cohort of 18065 eyes showing the cumulative retinal detachment rate (most retinal detachment events occur in the first 3 years). 

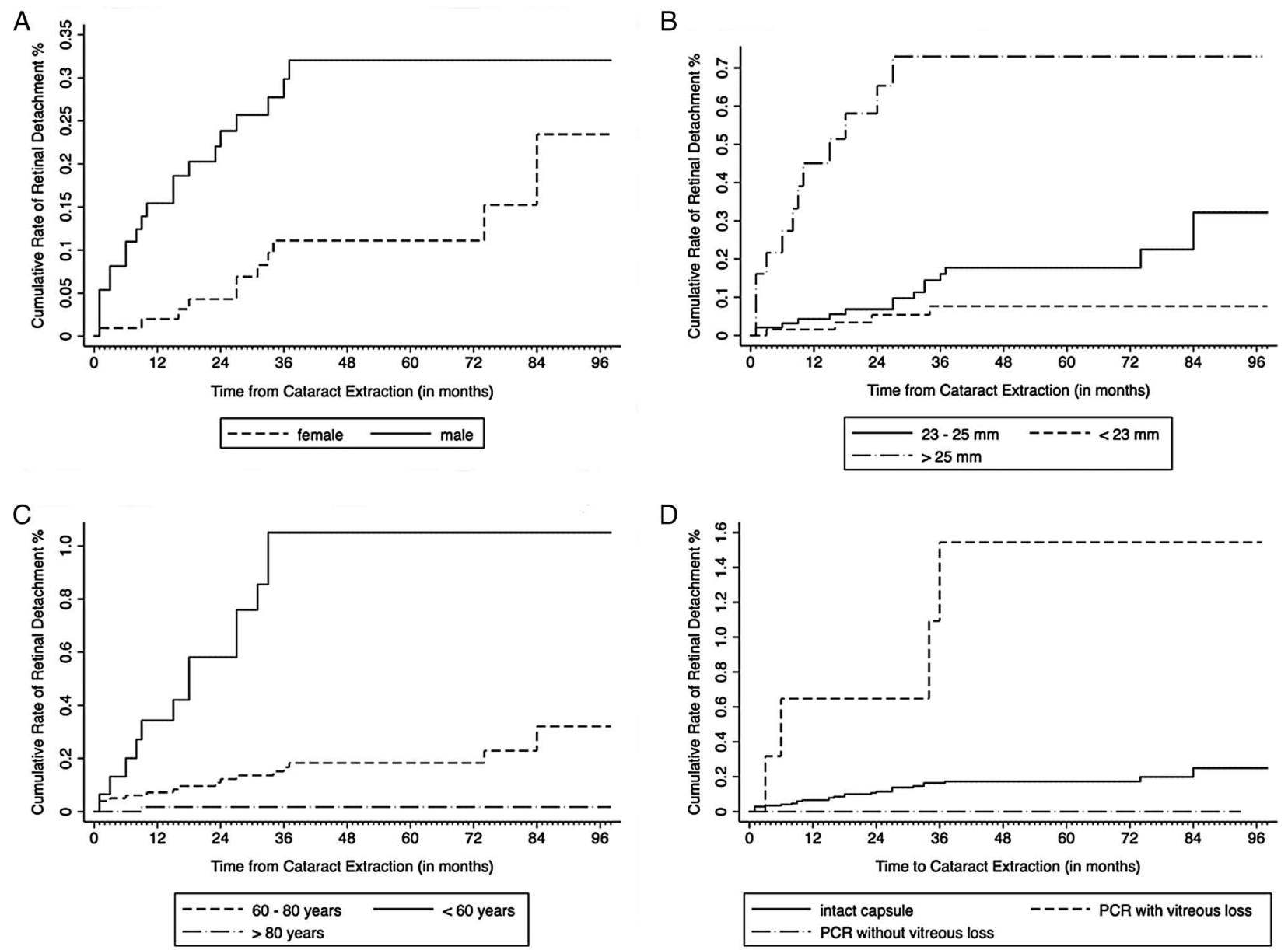

Figure 2 Cohort of 18065 eyes showing comparison of the cumulative retinal detachment rate: (A) by gender, (B) age, (C) axial length and (D) PCR. PCR, posterior capsular rupture.

\section{DISCUSSION}

This study looked at the incidence of RD after cataract surgery and associated risk factors in first operated eyes of 18065 patients in a geographically localised region in the UK. We found a cumulative rate of $\mathrm{RD}$ of $0.30 \%$ in 7 years and observed a fivefold increased risk in patients $<60$ years and almost a fourfold rise in eyes $>25 \mathrm{~mm}$ in length. PCR with vitreous loss was associated with a $>13$-fold increase in RD after cataract surgery.

The unique benefit of the methodology employed in this study is the $100 \%$ data capture of age, gender, axial length and the presence or absence of specified operative complications during cataract surgery, which should provide a reliable estimate of the relative risk of these factors on the development of subsequent RD.

As identified in previous studies, we found that male gender, younger age, longer axial length and intraoperative complications (PCR) during cataract surgery are all risk factors for the development of RD after cataract surgery. Male gender is a well established risk factor considered to increase the RD rate by 2.5 -fold. $^{2}{ }^{3}$ In our study, the respective HR was 2.0 (95\% CI 1.03 to 3.88 ) and was significant in the univariate model $(p=0.04)$ but not in the multivariate model $(p=0.43)$. This is probably because in our sample men were on average younger and had longer eyes than women (table 1). It is postulated that men were, therefore, less likely to have benefited from the protective effect of an age-related PVD while at the same time the longer axial length increased the risk for RD. It seems likely that for patients of the same age and with eyes of similar axial lengths, gender alone would not influence the risk of RD after cataract surgery.

Consistent with previous studies, we confirmed a strong effect of patient age on the risk of RD after cataract surgery significant in both univariate and multivariate models (table 2). Clark et al ${ }^{2}$ estimated a doubling of RD risk for every decade of younger age down to 50 years of age. However, the use of an extreme age group ( $>80$ years) and not the mean age group of patients as the reference group, results in dramatically high OR figures that could easily be misinterpreted. As postulated in previous studies the protective effect of advanced age is probably because the causative event for the vast majority of RDs is the development of a PVD and if this event has taken place before cataract surgery, the risk of subsequent RD is very low. ${ }^{15}$ The prevalence of PVD increases with age, from $40 \%$ in people aged $50-57$ years to $78.1 \%$ in people aged $65-72$ years. ${ }^{15}$ Ripandelli et al ${ }^{15}$ reported a $78.7 \%$ rate of PVD after cataract surgery during a follow-up between 2 days and 26 months (mean: 7.3 months) and Mirashahi observed a cumulative rate of $58.6 \%$ at 1 year. ${ }^{16}$ Various theories have been suggested to explain the high rate of PVD soon after cataract surgery including: alterations in the vitreous humour structure in pseudophakic eyes, and the lack of protuberance of the crystalline lens in pseudophakic patients that could increase the vitreous traction during the ocular saccades. ${ }^{17} 18$ The risk of RD after cataract surgery is much higher 




for eyes that developed a PVD postoperatively (7.4\%) compared with eyes with a PVD present before cataract surgery (1.1\%). For all the above reasons younger patients are at greater risk and older patients are relatively protected from the risk of RD after cataract surgery. ${ }^{15}$

Longer axial length is another established risk factor for RD that was confirmed in our study. ${ }^{8}$

This is the first study in the literature to possibly suggest a difference in subsequent RD risk in eyes with PCR with vitreous loss during cataract extraction compared with eyes with PCR without vitreous loss. Eyes with PCR and vitreous loss had a 13-16 times higher risk for RD compared with eyes with an intact capsule but there were no RD events among eyes with PCR and no vitreous loss $(n=72)$. All previous studies were only able to infer the presence of PCR with vitreous loss during surgery and could not distinguish between PCR with or without vitreous loss.9 121920 They confirmed a 14.8-18.8-fold higher risk for RD compared with eyes with an intact capsule. ${ }^{9} 21$ Ours is the only study of which we are aware, where the impact of the precise operative complication has been analysed. However, because the number of eyes with PCR without vitreous loss was small $(n=72)$, a larger sample size is needed to confirm whether such eyes have a different risk of subsequent RD. Another strength of this study, in common with the study of Lin et al, ${ }^{5}$ is that our analysis was limited to first operated eyes to avoid bias due to correlation between eyes of the same patient. ${ }^{2} 3$

The estimate of the absolute cumulative rate of RD in this study $(0.3 \%$ by 7 years) may be an underestimate of the true rate and is towards the lower end of that reported in the literature $\left(0.16 \%-2.31 \%\right.$ at $7-10$ years). ${ }^{1-5}$ This may be contributed to by lack of reliable capture of date of death data within the EMR as it is not automatically retrieved from the hospital administration system unless a record number is typed into the EMR and hence is not reliably recorded within the dataset we are able to extract. We report a $0.45 \%$ death rate in 7 years compared with $5.1 \%-21.4 \%$ for patients $>45-55$ years in other studies. ${ }^{22}{ }^{23}$ In addition, patients may have presented with RD to other units, but this factor was mitigated by searching the database of our closest neighbouring units. The rate of RD in our study may also reflect a truly reduced incidence of $\mathrm{RD}$ in the phacoemulsification compared with extracapsular cataract extraction (ECCE) era. Clark et $\mathrm{al}^{2}$ in a study of 65055 eyes operated between 1989 and 2001 reported a 10 -year RD rate of $0.68 \%$, but when comparing consecutive time periods he found a significant drop in RD rates from $0.96 \%$ between 1989 and 1993 to $0.25 \%$ for those operated between 1999 and 2001. Lin et $a l^{5}$ reported a 7 -year cumulative RD rate of $0.84 \%$ in 9184 eyes having cataract surgery from 2000 to 2010, but this study included ECCE and the female:male ratio was 1:2. Since ECCE and male gender are suspected to carry an increased risk for pseudophakic RD it is, therefore, not surprising that the rate they documented was higher than in our study. ${ }^{2}{ }^{6}$ By comparison the age-standardised incidence of RD in the community is around 11.2-17.9 cases per 100000 people per annum, making pseudophakic RD five times more likely than RD in the general population. ${ }^{24} 25$ Even if our absolute cumulative rate of $\mathrm{RD}$ is a slight underestimate of the true rate we believe there is no reason that the relative rates in different PCR groups should be biased by any of these limitations.

In conclusion, according to our results younger age, longer axial length and PCR with vitreous loss increase this risk for $\mathrm{RD}$ after cataract surgery. The data in this study though suggests that PCR without vitreous loss may not increase the risk of RD but a larger sample is needed to confirm or refute this observation. 
Contributors All authors had a substantial contribution to the conception, the design of the work, the acquisition and the analysis or interpretation of data. They all helped with drafting the work or revising it critically for important intellectual content. They all approved the final version sent to the journal for publishing and they agree to be accountable for all aspects of the work in ensuring that questions related to the accuracy or integrity of any part of the work are appropriately investigated and resolved.

Competing interests RLJ is a director and shareholder in Medisoft the electronic medical record (EMR) supplier that has its EMR installed at the hospitals from which data were extracted.

Ethics approval This is a retrospective data analysis study and was approved by clinical audit review board of the Gloucestershire Hospitals NHS Foundation Trust.

Provenance and peer review Not commissioned; externally peer reviewed.

\section{REFERENCES}

1 Quek DT-L, Lee SY, Htoon HM, et al. Pseudophakic rhegmatogenous retinal detachment in a large Asian tertiary eye centre: a cohort study. Clin Experiment Ophthalmol 2012;40:e1-7.

2 Clark A, Morlet N, Ng JQ, et al. Risk for retinal detachment after phacoemulsification: a whole-population study of cataract surgery outcomes. American Medical Association, 2012.

3 Boberg-Ans G, Henning V, Villumsen J, et al. Longterm incidence of rhegmatogenous retinal detachment and survival in a defined population undergoing standardized phacoemulsification surgery. Acta Ophthalmol Scand 2006;84:613-8.

4 Erie JC, Raecker MA, Baratz KH, et al. Risk of retinal detachment after cataract extraction, 1980-2004: a population-based study. Ophthalmology 2006;113:2026-32.

5 Lin J-Y, Ho W-L, Ger L-P, et al. Analysis of factors correlated with the development of pseudophakic retinal detachment-a long-term study in a single medical center. Graefes Arch Clin Exp Ophthalmol 2013;251:459-65.

6 Sheu S-J, Ger L-P, Ho W-L. Late increased risk of retinal detachment after cataract extraction. Am J Ophthalmol 2010;149:113-9.

7 Clark A, Morlet N, Ng JQ, et al. Whole population trends in complications of cataract surgery over 22 years in Western Australia. Ophthalmology 2011;118:1055-61.

8 Sheu S-J, Ger L-P, Chen J-F. Axial myopia is an extremely significant risk factor for young-aged pseudophakic retinal detachment in Taiwan. Retina (Philadelphia, Pa) 2006;26:322-7. http://www.ncbi.nlm.nih.gov/pubmed/16508433 (accessed 24 Dec 2014).

9 Jakobsson G, Montan P, Zetterberg M, et al. Capsule complication during cataract surgery: retinal detachment after cataract surgery with capsule complication:
Swedish Capsule Rupture Study Group report 4. J Cataract Refract Surg 2009;35:1699-705.

10 Royal College of Ophthalmologist. The Cataract National Data Set (CND). 2010. http://www.rcophth.ac.uk/core/core_picker/download.asp?id=854\&filetitle=Cataract +National+Data+set+submission (accessed 4 Feb 2015)

11 Royal College of Ophthalmologists. The Retinal Detachment Data Set. 2011. http:/l www.rcophth.ac.uk/core/core_picker/download.asp?id=1217\&filetitle=Retinal +Detachment+Data+Set (accessed 4 Feb2015).

12 Sparrow JM, Taylor H, Qureshi K, et al. The Cataract National Dataset electronic multi-centre audit of 55,567 operations: risk indicators for monocular visual acuity outcomes. Eye (Lond) 2012;26:821-6.

13 Jackson TL, Donachie PHJ, Sparrow JM, et al. United Kingdom National Ophthalmology Database Study of Vitreoretinal Surgery: report 1; case mix complications, and cataract. Eye (Lond) 2013;27:644-51.

14 Jackson TL, Donachie PHJ, Sallam A, et al. United Kingdom National Ophthalmology Database Study of Vitreoretinal Surgery: report 3, retinal detachment. Ophthalmology 2014;121:643-8.

15 Ripandelli G, Coppé AM, Parisi V, et al. Posterior vitreous detachment and retinal detachment after cataract surgery. Ophthalmology 2007;114:692-7.

16 Mirshahi A, Hoehn F, Lorenz K, et al. Incidence of posterior vitreous detachment after cataract surgery. J Cataract Refract Surg 2009;35:987-91.

17 Neal RE, Bettelheim FA, Lin C, et al. Alterations in human vitreous humour following cataract extraction. Exp Eye Res 2005;80:337-47.

18 HILDING AC. Alterations in the form, movement, and structure of the vitreous body in aphakic eyes. AMA Arch Ophthalmol 1954;52:699-709. http://www.ncbi.nlm. nih.gov/pubmed/13206500 (accessed 22 Feb 2015).

19 Jaycock P, Johnston RL, Taylor $\mathrm{H}$, et al. The Cataract National Dataset electronic multi-centre audit of 55,567 operations: updating benchmark standards of care in the United Kingdom and internationally. Eye (Lond) 2009;23:38-49.

20 Day AC, Donachie PHJ, Sparrow JM, et al. The Royal College of Ophthalmologists' National Ophthalmology Database study of cataract surgery: report 1, visual outcomes and complications. Eye (Lond) 2015;29:552-60.

21 Clark A, Morlet N, Ng JQ, et al. Long-term trends and outcomes of anterior vitrectomy in Western Australia. Acta Ophthalmol 2015;93:27-32.

22 Nucci C, Cedrone C, Culasso F, et al. Association between lens opacities and mortality in the Priverno Eye Study. Graefes Arch Clin Exp Ophthalmol 2004;242:289-94.

23 Borger PH, van Leeuwen $\mathrm{R}$, Hulsman CAA, et al. Is there a direct association between age-related eye diseases and mortality? The Rotterdam Study. Ophthalmology 2003;110:1292-6.

24 Rowe JA, Erie JC, Baratz KH, et al. Retinal detachment in Olmsted County, Minnesota, 1976 through 1995. Ophthalmology 1999;106:154-9.

25 Mitry D, Chalmers J, Anderson K, et al. Temporal trends in retinal detachment incidence in Scotland between 1987 and 2006. Br J Ophthalmol 2011;95:365-9. 